\title{
Analysis and Experimental Investigation of Vibration Characteristics of Rotary Platform of Hydraulic Excavator under Complex Working Conditions
}

\author{
Tinghao Li, ${ }^{1,2}$ Fuxiu Liu, ${ }^{1}$ Zhaojun Li $\oplus_{0},{ }^{1}$ Mingjin Lu, ${ }^{1}$ and Qiulu $\mathrm{He}^{1}$ \\ ${ }^{1}$ College of Mechanical Engineering, Guangxi University, Nanning 530004, China \\ ${ }^{2}$ Institute of Systems Engineering, Macau University of Science and Technology, Macau 999078, China \\ Correspondence should be addressed to Zhaojun Li; lizhaojun@gxu.edu.cn
}

Received 7 September 2021; Revised 19 October 2021; Accepted 6 November 2021; Published 3 December 2021

Academic Editor: Jiaqiang E.

Copyright (c) 2021 Tinghao Li et al. This is an open access article distributed under the Creative Commons Attribution License, which permits unrestricted use, distribution, and reproduction in any medium, provided the original work is properly cited.

\begin{abstract}
The rotary platform is the load-bearing substrate of a hydraulic excavator. The dynamic characteristics of the rotary platform directly affect the reliability and safety of the whole machine of a hydraulic excavator. In this work, the characteristics of the main external excitations acting on the hydraulic excavator such as the engine excitation, pressure pulsation excitation of the piston pump, inertial excitation of the working device, and road excitation are analyzed. The vibration transmission paths under the action of external excitations are ascertained. A vibration test method for the rotary platform of the hydraulic excavator is proposed. The vibration characteristics of the rotary platform under complex working conditions are researched, and the internal relationships between the vibration characteristics of the rotary platform and the engine excitation, pressure pulsation excitation of the piston pump, and road excitation are analyzed experimentally. The results show that the rotary platform is subjected to different excitations when it is under different working conditions. Moreover, the internal relationships between the dynamic characteristics of the rotary platform and the external excitation characteristics can be discovered by analyzing the vibration signals of the rotary platform, and the dynamic characteristics of the whole machine of the hydraulic excavator can be deeply studied based on the vibration characteristics of the rotary platform.
\end{abstract}

\section{Introduction}

Hydraulic excavators are widely used in many fields and play a very important role in reducing manual labor and improving labor production efficiency [1]. They are not only used in the field of mining [2] but also in earthwork construction, such as farmland transformation and demolition [3]. The hydraulic excavator is mainly composed of the rotary platform, cab, working device, engine, hydraulic system, and walking device. The main components are installed on the rotary platform of the hydraulic excavator, which means that the rotary platform is not only the loadbearing substrate of the hydraulic excavator but also an indispensable intermediate link in the vibration transmission path. Obviously, there is a coupling relationship between the rotating platform and the main devices of the hydraulic excavator, such as the power device, working device, and walking device. Thus, the dynamic performance of the rotary platform can effectively reflect the internal relationships between the rotary platform and other devices within the hydraulic excavator. Therefore, to ensure the safe and reliable operation of the hydraulic excavator, it is necessary to analyze the vibration characteristics of the rotary platform.

At present, research works on the dynamics of the rotary platform of hydraulic excavators have mainly focused on theoretical and simulation studies. For example, Li et al. [4] calculated the natural frequencies and mode shapes of the rotary platform of the hydraulic excavator and proposed a method to avoid the resonance of the rotary platform by modifying its structure. The result showed that the root mean square value of the vibration acceleration of the improved prototype cab is $0.12 \mathrm{~m} / \mathrm{s}^{2}$, which is lower than the value of $0.25 \mathrm{~m} / \mathrm{s}^{2}$ before improvement. Zhang et al. [5] 
found the weak points of the structure of the rotary platform of the hydraulic excavator using the finite element method, allowing its stability and overall performance to be enhanced. The result showed that the maximum composite stress in the fatigue failure area under all working conditions decreased from 172.46 $\mathrm{MPa}$ to $123.82 \mathrm{MPa}$. Yang et al. [6] established a coupled lateral-torsional vibration model of the rotary mechanism of the hydraulic excavator by considering the rotary platform as an equivalent rotational inertia unit. Jovanović et al. [7] developed software to enable the determination and detailed analysis of the slewing bearing load in the entire working range of the excavator. Qin et al. [8] proposed a hydraulic-electric hybrid excavator swing drive system. They verified that the energy consumption of the proposed system was reduced by $37.26 \% \sim 53.29 \%$ within a swing working cycle compared with the original system, and the backswing phenomenon of the swing system is suppressed. However, there have been few theoretical research studies on the vibration response of the rotary platform of a hydraulic excavator under complex working conditions.

Experimental investigations are an important method for studying the dynamic characteristics of mechanical structures. Therefore, increasing attention has focused on experimental research studies. For example, Wei et al. [9] undertook experiments to investigate the vibration response performances of a rotating beam with respect to the intensity of the electric field, rotation speed, and acceleration. They found that the vibration of the beam caused by the rotating motion at different rotating speeds and acceleration could be quickly suppressed by applying the electric field to the ER beam and evaluated the feasibility of ER fluid in attenuating the vibration of rotating beams. Zheng et al. [10] dealt with the modeling and analysis of random vibration tests with six degrees of freedom, involving an advanced multi-input/ multioutput system in which three translational and three rotational motions could be simultaneously controlled. Jiang et al. [11] provided an alternative method for testing the lowfrequency vibration of a huge bucket-wheel excavator and obtained accurate estimates of the low natural frequencies of the bucket-wheel excavator. Hydraulic excavators are a complex vibration system; therefore, experimental investigations are important to reveal their vibration characteristics. At present, experimental investigations of the vibration characteristics of hydraulic excavators are mainly focused on the vibration characteristics of the cab. For example, Alphin et al. [12] regarded whole-body vibration to be a major health hazard among operators, so they conducted field measurements of the whole-body vibration in tracked excavators with a breaker by using a standard vibration measurement system. The experimental result showed that the vibration dose value recorded was $17.6-62.72 \mathrm{~m} / \mathrm{s}^{1.75}$, and whole-body vibration exposure of the breaker operator was much higher and lies beyond the upper limit as given in ISO 2631-1. Lu and Zhou [13] processed the measured seat vibration responses of a hydraulic excavator and identified the vibration signals corresponding to different excitation sources through the time-frequency analysis method combined with ensemble empirical mode decomposition and a continuous wavelet transform. Wang et al. [14] proposed measures to improve the vibration characteristics of the cab following vibration testing of hydraulic excavators. The result showed that the vibration isolation performance of the improved prototype cab was obviously improved below $40 \mathrm{~Hz}$ and above $200 \mathrm{~Hz}$. Pang et al. [15] built an operational transfer path analysis model of vibration from the engine to the cab seat using the vibration test data of an excavator under working conditions. The result showed that the frequencydomain features of the synthesized output signal agree well with those of the test output signal; thus, the model is proved valid. Dąbrowski and Dziurdź [16] present the proposition of a simple estimation of noise and vibration propagation paths of the machine and verify the effectiveness of a proposed procedure on the basis of the analysis of hydraulic excavator. They found that this procedure helped to minimize the transfer of vibrations of power units in selected frequency ranges that led to the change of overall noise level in operator's cab about $5 \mathrm{~dB}$. However, few experimental studies have investigated the vibration characteristics of the rotary platform under complex working conditions.

In this study, the vibration characteristics of the rotary platform of a hydraulic excavator are tested and studied under complex working conditions. The aim of this study is to comprehensively analyze the influence of various excitations on the dynamic characteristics of the rotary platform and to provide a useful basis for discovering the coupling relationships between the rotary platform and other related devices of the hydraulic excavator.

The CLG906D tracked hydraulic excavator is taken as the research object in this study, as shown in Figure 1.

\section{Analysis of Vibration Characteristics of Rotary Platform}

2.1. Vibration Characteristic. Using the finite element method, the dynamic equation of the rotary platform of the hydraulic excavator can be established in the form:

$$
\mathbf{M U}+\mathbf{C} \dot{U}+\mathrm{KU}=\mathbf{F},
$$

where $\mathbf{M}, \mathbf{K}$, and $\mathbf{C}$ are the mass matrix, stiffness matrix, and damping matrix of the rotary platform of hydraulic excavator, respectively; $\ddot{\mathbf{U}}, \dot{\mathbf{U}}$, and $\mathbf{U}$ are the generalized acceleration vector, generalized velocity vector, and generalized displacement vector, respectively; $\mathbf{F}$ is the excitation acting on the hydraulic excavator, including the engine excitation, pressure pulsation excitation of the piston pump, inertial excitation of the working device, and road excitation [17].

According to the dynamic equation (1), the frequency equation of the rotary platform of the hydraulic excavator can be expressed as

$$
\left|\mathbf{K}-\omega^{2} \mathbf{M}\right|=0
$$

where $\omega$ is the natural frequency of the rotary platform of hydraulic excavator.

According to equation (2), the natural frequencies of the rotary platform of the hydraulic excavator can be calculated and the modes of the rotary platform of the hydraulic excavator can also be determined. 


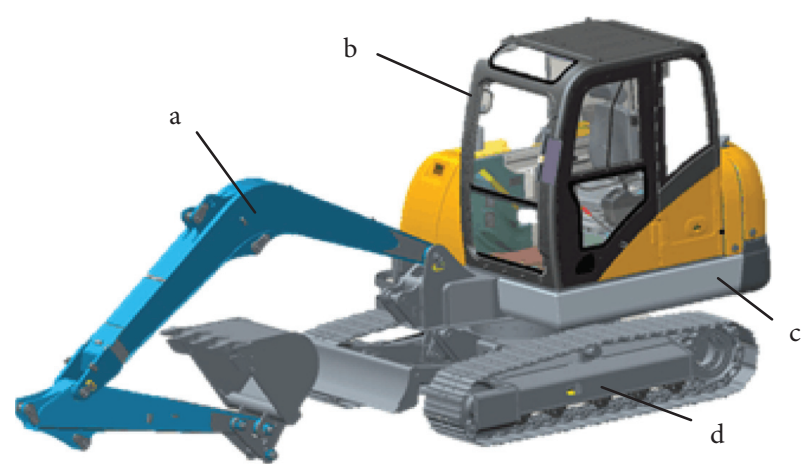

Figure 1: CLG906D tracked hydraulic excavator. (a) Working device. (b) Cab. (c) Rotary platform. (d) Walking device.

According to the dynamic equation (1), the dynamic response of the rotary platform is calculated by the modal superposition method, which can be expressed as

$$
\mathbf{U}=\sum_{i=1}^{N} \eta_{i}(t) \varphi^{(i)}
$$

where $\varphi^{(i)}$ is the $i$-th order modal vector of the regular modal matrix; $\eta_{i}(t)$ is the response of the rotary platform under the $i$-th canonical coordinate; and $N$ is the number of degrees of freedom of the rotary platform.

2.2. Analysis of Main Excitations. Under complex working conditions, the excitations affecting a hydraulic excavator are very complicated. The excitations that impact a hydraulic excavator mainly include the following: the engine excitation, the pressure pulsation excitation of the piston pump, the inertial excitation of the working device, and the road excitation.

2.2.1. Engine Excitation. The engine excitation of a hydraulic excavator mainly comes from the internal cylinder gas pressure during engine operation and the unbalanced inertia forces and moments of inertia caused by the moving mass. For an in-line four-cylinder Yanmar engine, as is often used in hydraulic excavators, the second-order unbalanced inertia force $F_{1}$ can be expressed as [18]

$$
F_{1}=-4 m_{b} \lambda R_{w}\left(\pi f_{F 1}\right)^{2} \cos \left(f_{F 1} t\right),
$$

where $m_{b}$ is the mass of the moving parts; $R_{w}$ is the crank radius; $\lambda=R_{w} / l, l$ is the rod length; $t$ is the time; and $f_{F 1}$ is the frequency of the engine excitation $(\mathrm{Hz})$ and can be expressed as

$$
f_{F 1}=\frac{n_{F}}{30},
$$

where $n_{F}$ is the engine speed.

2.2.2. Pressure Pulsation Excitation of Piston Pump. The piston pump is an important part of the hydraulic system of hydraulic excavator. When a piston pump operates, the pressure pulsation excitation occurs [19]. This is one of the main sources of vibration and noise for the hydraulic excavator. According to the working principle of the piston pump, the pressure pulsation excitation of the piston pump $F_{2}$ can be expressed as [20]

$$
F_{2}=A_{K}\left(B_{0}+\sum_{n=1}^{\infty} B_{n} \sin \left(n f_{F 2} t+\psi_{n}\right)\right),
$$

where $A_{K}$ is the area of the bottom surface of the plunger, $B_{0}$ is the pressure pulsation constant term of the piston pump, $B_{n}$ is the pressure pulsation amplitude of the piston pump, $\psi_{n}$ is the pressure pulsation phase angle of the piston pump, and $B_{0}, B_{n}$, and $\psi_{n}$ are related to the structural parameters and material parameters of the piston pump, which can be obtained from reference [20]; $f_{F 2}$ is the pressure pulsation excitation frequency of the piston pump $(\mathrm{Hz})$ and can be expressed as

$$
f_{F 2}=\frac{z n_{F}}{60},
$$

where $z$ is the number of pistons in the piston pump.

2.2.3. Inertial Excitation of Working Device. In the working process of the hydraulic excavator, the working device often starts and stops. Thus, the working device will generate inertial excitations [21]. As the working device is installed on the rotary platform of the hydraulic excavator, the inertial excitation of the working device is transmitted to the rotary platform through the fixed devices. According to reference [22], the inertial excitation of the working device $F_{3}$ can be expressed as

$$
F_{3}=m_{c} \frac{\Delta v}{T_{c}}(1+\tau)\left(1-\cos f_{F 3} t\right),
$$

where $m_{c}$ is the mass of the working device in the excitation process; $\Delta v$ is the velocity variation in the excitation process; $T_{c}$ is the excitation time; $\tau$ is the excitation recovery coefficient; and $f_{F 3}$ is the inertial excitation frequency of the working device $(\mathrm{Hz})$ and can be expressed as

$$
f_{F 3}=\frac{2 \pi}{T_{c}}
$$

2.2.4. Road Excitation. The excavator considered in this study is a tracked hydraulic excavator. When the tracked hydraulic excavator moves along an uneven road, the road excitation produces a large low-frequency vibration. Thus, it is necessary to analyze the influence of road excitation on the vibration characteristics of the tracked hydraulic excavator [23].

Let $Z(x)$ be the pavement unevenness function for a pavement length of $x$. When the tracked hydraulic excavator is traveling at a speed of $v$, the road excitation can be expressed as [24]

$$
s(t)=Z(v t),
$$

where $x=v t$ and $t$ is the time; the vertical road excitation frequencies are mainly distributed in the range $8-10 \mathrm{~Hz}$ and integer multipliers of this frequency range [24]. 
2.3. Analysis of Vibration Transmission Path. The essence of vibration transfer is the transfer of energy [25]. To distinguish the vibration characteristics under different excitations, it is necessary to analyze the vibration transmission paths under different excitation types.

The engine is the power source of the hydraulic excavator and the piston pump. The engine output shaft end and the piston pump input shaft end are connected by a rigid coupling, so the engine and the piston pump can be taken as a single system when analyzing the vibration transmission paths. The system composed of the engine and the piston pump is named the engine-piston pump system. The engine-piston pump system has two excitations, namely, engine excitation and pressure pulsation excitation of the piston pump. According to the working principle and structural characteristics of the hydraulic excavator, the engine is fixed on the rotary platform by the connecting bolts. One end of the piston pump shaft is connected to the engine output shaft by the coupling, and the other end of the piston pump shaft is joined by the pipe joint to the hydraulic hoses that are linked with the hydraulic components and the fixed devices [26], that is, the hydraulic components and the fixed devices are directly or indirectly connected with the rotary platform. Therefore, the engine excitation and the pressure pulsation excitation of the piston pump produced by the engine-piston pump system are transmitted to the rotary platform through two paths: (i) the engine excitation and the pressure pulsation excitation of the piston pump are transmitted to the rotary platform through the engine connecting bolts; (ii) the excitations are transmitted to the rotary platform through the hydraulic components and the fixed devices.

When the working device starts and stops, the inertial excitation of the working device can be transmitted to the rotary platform through the fixed devices. This is because the working device is installed on the rotary platform of the hydraulic excavator by the fixed devices. Similarly, according to the structural characteristics of the hydraulic excavator, when the tracked hydraulic excavator moves along an uneven road, the road excitation is transmitted to the rotary platform through the guide wheel, support wheel, driving wheel, and so on. In summary, the main vibration transmission paths of the rotary platform of the hydraulic excavator are shown in Figure 2.

2.4. Analysis of Vibration Transfer Characteristics. Using equation (1), the vibration characteristics of the rotary platform of the hydraulic excavator can be studied. By the Fourier transform of dynamic equation (1), the corresponding displacement frequency response function can be obtained [27]. The rotary platform of the hydraulic excavator is generally a steel structure. For steel structures, the amplitudes of the frequency response function decrease as the distance from the source increases. The location of the excitation source is recorded as $x_{0}$, and the amplitude of the frequency response function at the excitation source is recorded as $U\left(x_{0}\right)$. Thus, the amplitude of the frequency response function at a distance of $x$ from the excitation source can be expressed as [28]

$$
U(x)=U\left(x_{0}\right) e^{-\beta x},
$$

where $\beta$ is the attenuation coefficient.

Equation (11) implies that increasing the distance between the measuring point on the rotary platform and the excitation source will produce a smaller amplitude of the frequency response function and enhance the amplitude of vibration attenuation.

2.5. Modal Simulation. The vibration modes of the rotary platform of the hydraulic excavator can be analyzed by using the ANSYS Workbench software. The three-dimensional model of the rotary platform is shown in Figure 3. Here, the first four modes of the rotary platform are analyzed by simulation. The simulation results of the first four-order natural frequencies of the rotary platform are listed in Table 1.

\section{Vibration Test of Rotary Platform}

3.1. Vibration Test System. Electric measurement methods have many advantages, such as a wide range of testing frequencies and high sensitivity [29]. Thus, an electric measurement method was adopted to collect the vibration signals of the rotary platform. The vibration test site is shown in Figure 4. The instruments used in the vibration test include an ICP three-way acceleration sensor, LMS Test.LAB signal collector, and a notebook computer.

3.2. Measuring Points. The vibration characteristics of measuring point 1 and measuring point 2 on the rotary platform were analyzed experimentally. Measuring point 1 is located at the connection between the engine and the rotary platform, and measuring point 2 is located below the lefthand door of the cab, as shown in Figure 5. Obviously, measuring point 2 is farther away from the engine-piston pump system (the excitation source) than measuring point 1.

3.3. Vibration Signal Acquisition and Processing. In this study, the vibration signals of the rotary platform were collected by an ICP three-way acceleration sensor. According to the sampling theorem, the sampling frequency is selected to be $2,000 \mathrm{~Hz}$ because the vibration signals of the rotary platform are generally concentrated below $1,000 \mathrm{~Hz}$.

The frequency-domain analysis method is often used for vibration signal processing because the frequency-domain signals are more intuitive than time-domain signals [30]. As the vibration signals acquired by the acceleration sensor are usually time-domain signals, the FFT transformation function in the LMS Test.Lab system was used to convert the acquired time-domain signals into frequencydomain signals for the analysis of the vibration characteristics. 


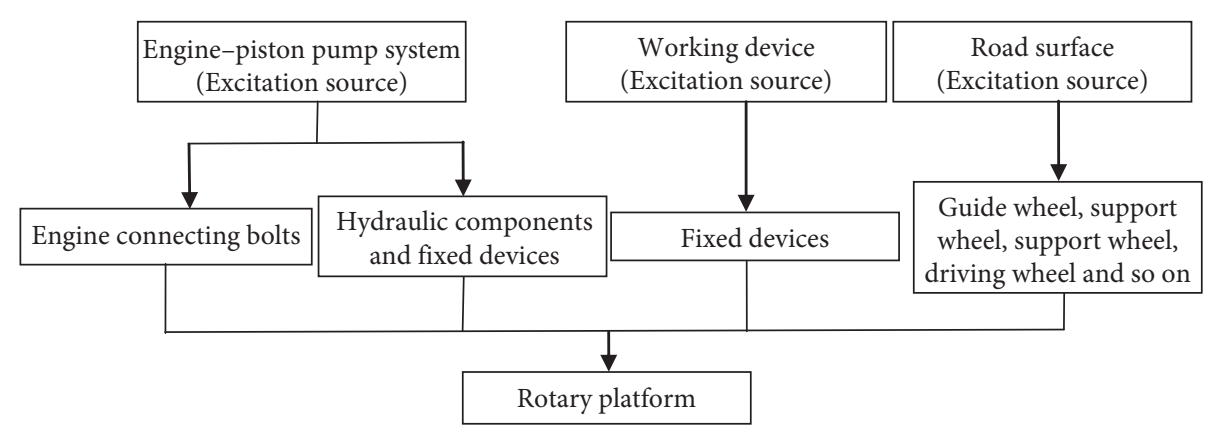

FIGURE 2: Vibration transmission paths of the rotary platform of hydraulic excavator.

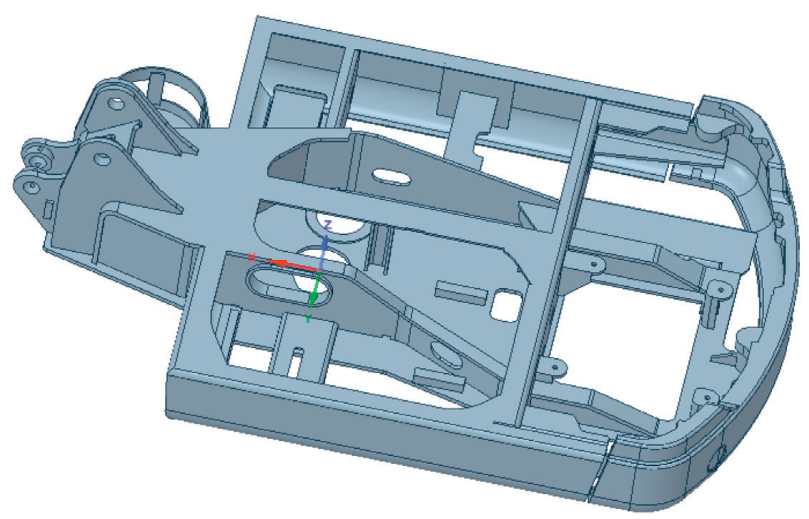

Figure 3: Three-dimensional model of the rotary platform.

TABLE 1: Simulation results of the first four-order natural frequencies of the rotary platform.

\begin{tabular}{lcc}
\hline Order & Frequency $(\mathrm{Hz})$ & Mode shape \\
\hline 1 & 10.06 & Bending \\
2 & 15.43 & Torsion \\
3 & 16.52 & Torsion \\
4 & 26.21 & Bending \\
\hline
\end{tabular}

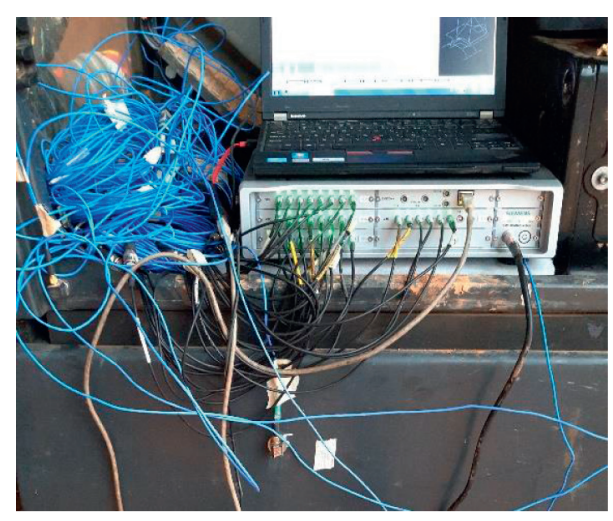

Figure 4: Vibration test site diagram.

\section{Analysis of Vibration Test Results of Rotary Platform}

4.1. Vibration Test under Fixed Collecting Bucket Condition. The fixed collecting bucket condition provides a modal test of the rotary platform of the hydraulic excavator. Under the

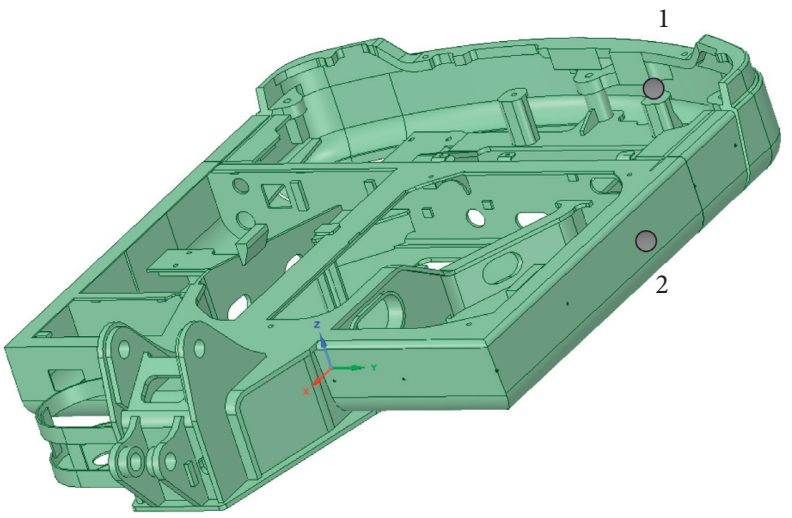

FIgURE 5: Measuring point positions on the rotary platform.

fixed collecting bucket condition, the working device of the hydraulic excavator is taken back and the collecting bucket test is carried out. As the working device is installed on the rotary platform of the hydraulic excavator, the rotary platform will be impacted by the inertial excitation of the working device as it retracts, which means that the rotary platform is subjected to a hammer excitation. Therefore, the impact on the rotary platform due to the retraction of the working device is equivalent to a swept-sine vibration test of the rotary platform. In this case, the vibration test under the fixed collecting bucket condition can be regarded as a modal test of the rotary platform.

According to the test data, the first four-order natural frequencies of the rotary platform are obtained as shown in Table 2. By comparing the test results of the first four-order natural frequencies of the rotary platform with the simulation results of the first four natural frequencies of the rotary platform (see Table 1), it can be seen that the modal simulation results are basically consistent with the test results. Therefore, it is feasible to obtain the modal of the rotary platform by experiments under the fixed collecting bucket condition. This provides a novel idea for testing the modal of the rotary platform of hydraulic excavator.

4.2. Vibration Test under Fixed Idle Speed Condition. According to the working principle and structural characteristics of the hydraulic excavator, when it is under the fixed idle speed condition, the engine is in the working state while the hydraulic system is not working. Thus, the excitation 
TABLE 2: Test results of the first four-order natural frequencies of the rotary platform.

\begin{tabular}{lcc}
\hline Order & Frequency $(\mathrm{Hz})$ & Mode shape \\
\hline 1 & 10.16 & Bending \\
2 & 15.31 & Torsion \\
3 & 16.23 & Torsion \\
4 & 26.06 & Bending \\
\hline
\end{tabular}

acting on the hydraulic excavator is mainly engine excitation, and the excitation source is the engine-piston pump system.

In Figure 5, measuring point 1 is located between the engine and the rotary platform, and measuring point 2 is the point on the rotary platform located below the lefthand door of the cab. Measuring point 2 is farther away from the engine-piston pump system (the excitation source) than measuring point 1 . Therefore, according to the vibration characteristics of measuring points 1 and 2 , the vibration characteristics of the rotary platform excited by the engine excitation can be studied and the vibration transmission characteristics of the rotary platform can be analyzed.

Under the fixed idle speed condition, the engine speed is $n_{F}=1050 \mathrm{r} / \mathrm{min}$, and according to equation (5), the engine excitation frequency is $f_{F 1}=35 \mathrm{~Hz}$. The vibration signals along the direction perpendicular to the rotary platform were obtained under the fixed idle speed condition. The experimental frequency-domain dynamic response characteristic curves of measuring points 1 and 2 under the fixed idle speed condition are shown in Figures 6 and 7, respectively. It can be seen from Figures 6 and 7 that there are obvious vibration peaks at the engine excitation frequency $(35 \mathrm{~Hz})$. The experimental results show that the rotary platform is excited by the engine excitation under the fixed idle speed condition, which is consistent with the theoretical analysis.

According to the experimental frequency-domain dynamic response characteristic curves in Figures 6 and 7, the values of the vibration peaks corresponding to the engine excitation frequency at measuring points 1 and 2 are listed in Table 3. It can be seen from Table 3 that the vibration peak value at measuring point 2 is $2.12 \%$ of that at measuring point 1 . The main reason why the vibration peak value at measuring point 2 is smaller than that at measuring point 1 is that measuring point 2 is farther away from the enginepiston pump system than measuring point 1. According to the vibration transmission characteristics, measuring points farther away from the excitation source will observe greater vibration attenuation.

The above results showed that the vibration signals of the rotary platform revealed the inherent relationship between the vibration characteristics of the rotary platform and the engine excitation under the fixed idle speed condition. Thus, the dynamic characteristics of other related devices of the hydraulic excavator can be analyzed based on the vibration characteristics of the rotary platform because the rotary platform is the indispensable intermediate link in the vibration transmission path of a hydraulic excavator.

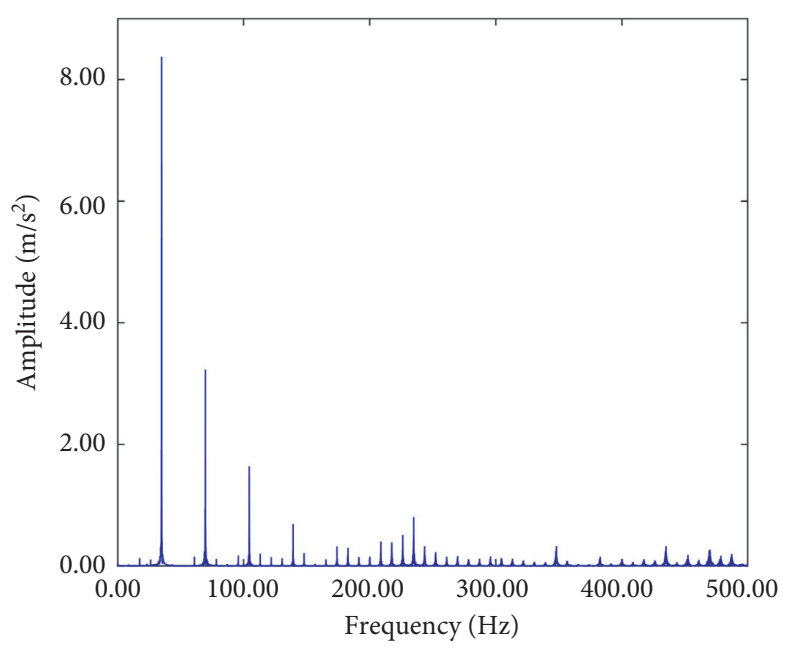

FIGURE 6: Experimental frequency-domain dynamic response characteristic curves of measuring point 1 in the vertical direction.

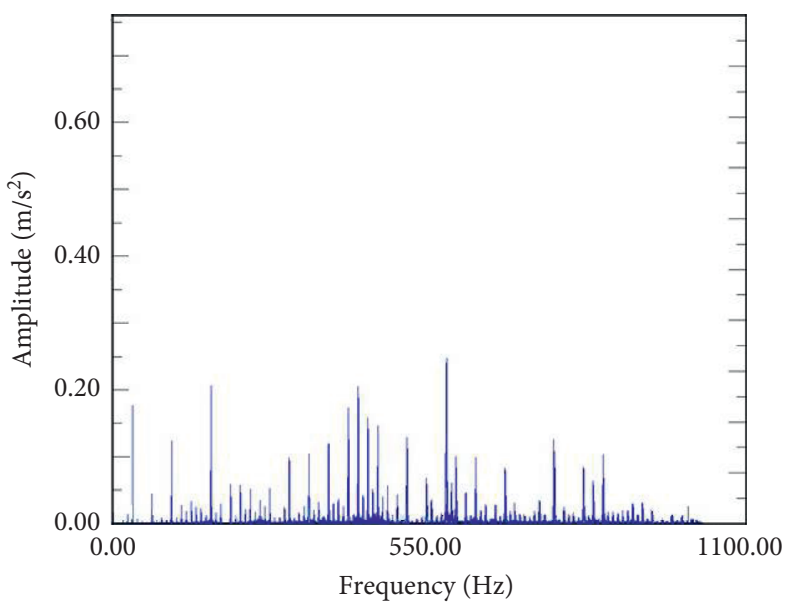

FIgURE 7: Experimental frequency-domain dynamic response characteristic curves of measuring point 2 in the vertical direction.

4.3. Vibration Test under Fixed Working Condition. According to the working principle and structural characteristics of the hydraulic excavator, under the fixed working condition, both the engine and the hydraulic system are in the working state. Therefore, the excitations acting on the hydraulic excavator are mainly the engine excitation and the pressure pulsation excitation of the piston pump under the fixed working condition, that is, the sources of excitations are all from the engine-piston pump system.

In Figure 5, measuring points 1 and 2 are on the rotary platform of the hydraulic excavator, and measuring point 2 is farther away from the excitation source than measuring point 1 . Therefore, based on the vibration characteristics of measuring points 1 and 2, the vibration characteristics of the rotary platform simultaneously excited by the engine excitation and the pressure pulsation excitation of the piston pump can be studied and the vibration transmission characteristics of the rotary platform can be analyzed.

Under the fixed working condition, the engine speed is $n_{F}=2250 \mathrm{r} / \mathrm{min}$; according to equation (5), the engine 
TABLE 3: Comparative analysis of vibration test results under fixed idle speed condition.

\begin{tabular}{lcc}
\hline Measuring point & Frequency corresponding to vibration peak $(\mathrm{Hz})$ & Vibration peak value $\left(\mathrm{m} / \mathrm{s}^{2}\right)$ \\
\hline 1 & 34.81 & 8.51 \\
2 & 35.46 & 0.18 \\
\hline
\end{tabular}

excitation frequency is $f_{F 1}=75 \mathrm{~Hz}$. As the number of pistons in the plunger pump is $z=10$, equation (7) implies that the pressure pulsation excitation frequency of the piston pump is $f_{F 2}=375 \mathrm{~Hz}$.

The vibration signals along the direction perpendicular to the rotary platform were obtained by the experiments under the fixed working condition. The experimental frequency-domain dynamic response characteristic curves of measuring points 1 and 2 under the fixed working condition of constant speed rotation are shown in Figures 8 and 9, respectively. It can be seen from Figures 8 and 9 that there are obvious vibration peaks at the pressure pulsation excitation frequency $(375 \mathrm{~Hz})$ and the engine excitation frequency $(75 \mathrm{~Hz})$. The experimental results show that the rotary platform is simultaneously excited by the engine excitation and the pressure pulsation excitation under the fixed working condition, which is consistent with the theoretical analysis results.

According to the experimental frequency-domain dynamic response characteristic curves in Figures 8 and 9, the values of vibration peaks corresponding to the pressure pulsation excitation frequency $(375 \mathrm{~Hz})$ and the engine excitation frequency $(75 \mathrm{~Hz})$ at measuring points 1 and 2 are listed in Table 4. It can be seen from Table 4 that the vibration peak value corresponding to the pressure pulsation excitation frequency $(375 \mathrm{~Hz})$ at measuring point 2 is $67.13 \%$ of that at measuring point 1 , that is, when the vibration excited by the pressure pulsation excitation of the piston pump is transmitted from measuring point 1 to measuring point 2, the vibration amplitude suffers an attenuation of $32.87 \%$. Moreover, the vibration peak value corresponding to the engine excitation frequency $(75 \mathrm{~Hz})$ at measuring point 2 is $22.92 \%$ of that at measuring point 1 , that is, the vibration excited by the engine excitation suffers an attenuation of $77.08 \%$ from measuring point 1 to measuring point 2.

According to the above results, the rotary platform is simultaneously excited by the engine excitation and the pressure pulsation excitation under the fixed working condition. When different excitations are acting on the rotary platform, the vibration characteristics and the vibration transfer characteristics of the rotary platform are not identical. Moreover, the above results also showed that the vibration signals of the rotary platform revealed the inherent relationship between the vibration characteristics of the rotary platform and the engine excitation and pressure pulsation excitation of the piston pump under the fixed working condition. Because the rotary platform is the loadbearing substrate of the hydraulic excavator, the dynamic characteristics of other related devices can be studied by analyzing both the vibration characteristics of the rotary platform and the coupling relationship between the rotating platform and other devices of the hydraulic excavator.

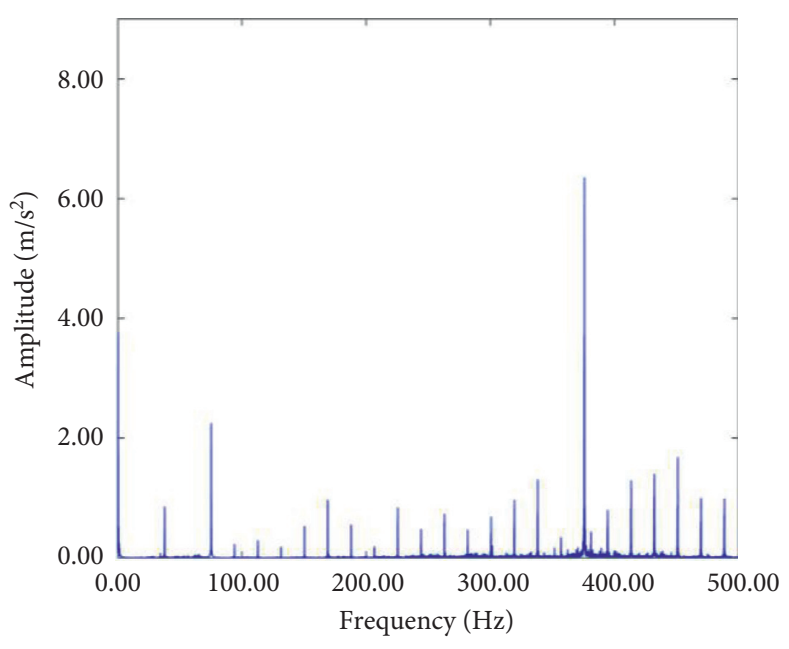

FIGURE 8: Experimental frequency-domain dynamic response characteristic curves of measuring point 1 in the vertical direction.

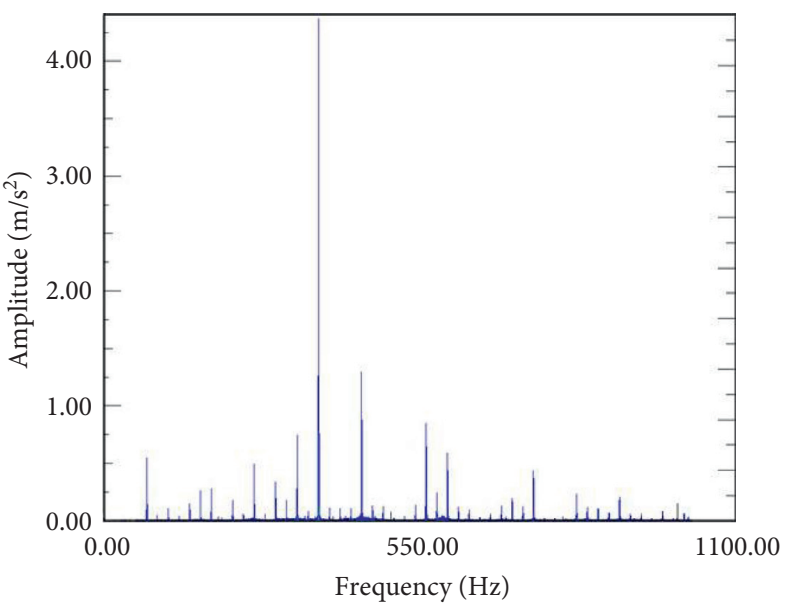

FIgURE 9: Experimental frequency-domain dynamic response characteristic curves of measuring point 2 in the vertical direction.

4.4. Vibration Test under Walking Condition. According to the working principle and structural characteristics of the hydraulic excavator, when the hydraulic excavator is under the walking condition, both the engine and the hydraulic system are in the working state, that is, under the walking condition, the hydraulic excavator is not only subject to the road excitation but also receives the engine excitation and the pressure pulsation excitation of the piston pump. Thus, the vibration characteristics of the rotary platform will be very complicated.

The vibration transmission characteristics of the rotary platform of the hydraulic excavator have been analyzed in Sections 4.2 and Sections 4.3 under the fixed idling conditions and the fixed working conditions, respectively. Here, 
TABLE 4: Comparative analysis of vibration test results under fixed working condition.

\begin{tabular}{lcr}
\hline Measuring point & Frequency corresponding to vibration peak $(\mathrm{Hz})$ & Vibration peak value $\left(\mathrm{m} / \mathrm{s}^{2}\right)$ \\
\hline \multirow{2}{*}{1} & 375.86 & 6.51 \\
& 75.14 & 2.40 \\
\hline \multirow{2}{*}{2} & 374.49 & 4.37 \\
& 74.99 & 0.55 \\
\hline
\end{tabular}

based on the experimental vibration signals at measuring point 2, the vibration characteristics of the rotary platform are analyzed when the rotary platform is simultaneously excited by the road excitation, the engine excitation, and the pressure pulsation excitation of the piston pump.

Under the walking condition, the engine speed is $n_{F}=2250 \mathrm{r} / \mathrm{min}$; according to equation (5), the engine excitation frequency is $f_{F 1}=75 \mathrm{~Hz}$. As the number of pistons in the piston pump is $z=10$, equation (7) gives the pressure pulsation excitation frequency to be $f_{F 2}=375 \mathrm{~Hz}$. Moreover, the road excitation must be considered when the hydraulic excavator is under the walking condition. According to equation (10) and reference [24], the road excitation frequencies are mainly distributed in the range $8-10 \mathrm{~Hz}$ and its integer multipliers.

The vibration signals along the direction perpendicular to the rotary platform were obtained by experiments under the walking condition. The experimental frequency-domain dynamic response characteristic curves of measuring point 2 under the rabbit speed walking condition is shown in Figure 10. It can be seen that there is an obvious vibration peak near the pressure pulsation excitation frequency $(375 \mathrm{~Hz})$, where the peak value is $1.77 \mathrm{~m} / \mathrm{s}^{2}$, and there is a vibration peak near the engine excitation frequency $(75 \mathrm{~Hz})$, where the peak value is $0.68 \mathrm{~m} / \mathrm{s}^{2}$, that is, the rotary platform of the hydraulic excavator is affected by the pressure pulsation excitation of the piston pump and the engine excitation. Figure 10 also indicates that there are very dense frequencies in the low-frequency range of the vibration signal, and these are mainly caused by road excitation. According to these results, it is clear that under the walking condition, the hydraulic excavator is not only excited by the road excitation but also by the engine excitation and the pressure pulsation excitation of the piston pump. Moreover, the above results also showed that the vibration signals of the rotary platform revealed the inherent relationship between the vibration characteristics of the rotary platform and the engine excitation, pressure pulsation excitation of the piston pump, and road excitation under the walking condition. Thus, according to the vibration characteristics of the rotary platform, the dynamic characteristics of other related devices can also be analyzed.

In summary, under complex working conditions such as a fixed idle speed, fixed working conditions, and walking conditions, the internal relationships between the vibration characteristics of the rotary platform and the external excitation characteristics can be analyzed by observing the vibration signals of the rotary platform. As the rotary platform is the indispensable intermediate link in the vibration transmission path of a hydraulic excavator, the dynamic characteristics of the whole machine of the hydraulic excavator can be effectively analyzed based on the

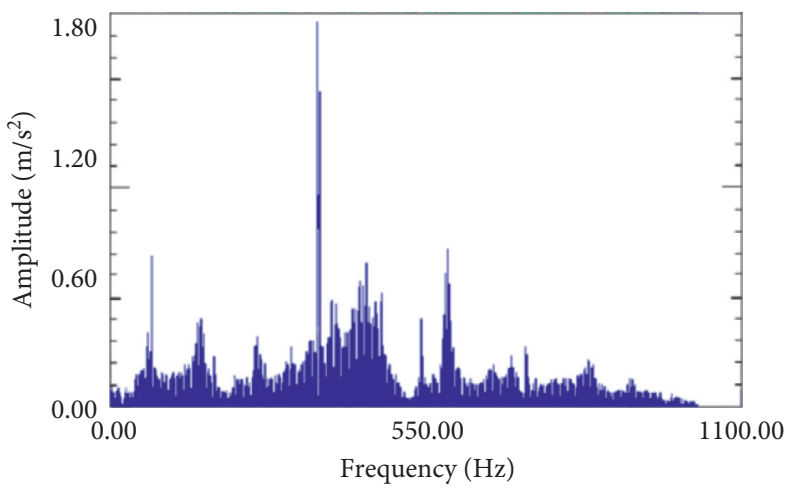

FIGURE 10: Experimental frequency-domain dynamic response characteristic curves of measuring point 2 in the vertical direction.

vibration characteristics of the rotary platform. This provides a new idea for deeply studying the dynamic characteristics of the whole machine of the hydraulic excavator under complex working conditions.

\section{Conclusions}

The hydraulic excavator was taken as the research object in this study. The vibration characteristics of the rotary platform of the hydraulic excavator were studied theoretically and experimentally. The studies show that

(1) The vibration of the rotary platform can be excited effectively by the inertial excitation of the working device as it retracts, which is equivalent to a sweptsine vibration test of the rotary platform. By the vibration test, the first four natural frequencies of the rotary platform are obtained, and the results are verified by the corresponding simulation results. It is found that the vibration test under the fixed collecting bucket condition can provide a novel and effective method for testing the modal of the rotary platform.

(2) The vibration signals of the rotary platform revealed the inherent relationship between the vibration characteristics of the rotary platform and the main excitations under complex working conditions. Moreover, the dynamic characteristics of other related devices can be studied by analyzing both the vibration characteristics of the rotary platform and the coupling relationship between the rotating platform and other devices of the hydraulic excavator.

(3) Under the fixed working condition, the vibration peak values at measuring points 1 and 2 that are 
excited by the pressure pulsation excitation of the piston pump are $6.51 \mathrm{~m} / \mathrm{s}^{2}$ and $4.37 \mathrm{~m} / \mathrm{s}^{2}$, respectively, and the corresponding vibration peak values that are excited by the engine excitation are $2.40 \mathrm{~m} / \mathrm{s}^{2}$ and $0.55 \mathrm{~m} / \mathrm{s}^{2}$, respectively. The results show that the influence of the pressure pulsation excitation of the piston pump on the dynamic characteristics of the rotary platform is much greater than that of engine excitation under the fixed working condition.

\section{Data Availability}

The data used to support the findings of this study are available from the corresponding author upon request.

\section{Conflicts of Interest}

The authors declare that they have no conflicts of interest.

\section{Acknowledgments}

This research was supported by the National Natural Science Foundation of China under grant no. 51465001.

\section{References}

[1] H. Ding, L. Han, W. Yang, and C. Wu, "Kinematics and dynamics analyses of a new type face-shovel hydraulic excavator," Proceedings of the Institution of Mechanical Engineers, Part C: Journal of Mechanical Engineering Science, vol. 231, no. 5, pp. 909-924, 2017.

[2] Y. Li, X. Mu, and R. Fan, "Multi-objective optimization and simulation of novel working mechanism for face-shovel excavator," International Journal of Intelligent Robotics and Applications, vol. 5, no. 1, pp. 1-9, 2021.

[3] F. Morosi, M. Rossoni, and G. Caruso, "Coordinated control paradigm for hydraulic excavator with haptic device," $A u$ tomation in Construction, vol. 105, Article ID 102848, 2019.

[4] Z. Li, W. P. Ding, W. Ding, R. Song, and X. C. Xu, "NVH improvement of hydraulic excavator based on modal analysis of rotary platform," Coal Mine Machinery, vol. 33, no. 1, pp. 191-193, 2012.

[5] W. Zhang, Q. He, and H. B. Wang, "Finite element analysis and improvement of the revolving platform of hydraulic excavator," Journal of Xuzhou Institute of Technology (Natural Sciences Edition), vol. 28, no. 3, pp. 64-68, 2013.

[6] X. J. Yang, G. H. Xu, Z. J. Li, and R. G. Wang, "Dynamic modeling and response analysis of lateral-torsional coupling vibration of the slewing mechanism of a hydraulic excavator," Advanced Materials Research, vol. 753-755, pp. 1755-1759, 2013.

[7] V. Jovanović, D. Janošević, and N. Petrović, "Analysis of slewing bearing load of a rotating platform drive in hydraulic excavators," Technical Gazette, vol. 21, no. 2, pp. 263-270, 2014.

[8] T. Qin, L. Ge, W. N. Huang, and L. You, "Operation characteristics and energy efficiency of swing drive system of hydraulic-electric hybrid excavator," Machine Tool \& $\mathrm{Hy}$ draulics, vol. 49, no. 8, pp. 111-116, 2021.

[9] K. Wei, G. Meng, S. Zhou, and J. Liu, "Vibration control of variable speed/acceleration rotating beams using smart materials," Journal of Sound and Vibration, vol. 298, no. 4-5, pp. 1150-1158, 2006.
[10] R. Zheng, H. Chen, A. Angeli, and D. Vandepitte, "A simplified modelling and analysis of six degree of freedom random vibration test," Mechanical Systems and Signal Processing, vol. 150, Article ID 107304, 2021.

[11] Y. Z. Jiang, C. J. Liu, X. J. Li, K. F. He, and D. M. Xiao, "Lowfrequency vibration testing of huge bucket wheel excavator based on step-decay signals," Shock and Vibration, vol. 2018, Article ID 6182156, 7 pages, 2018.

[12] M. S. Alphin, K. Sankaranarayanasamy, and S. P. Sivapirakasam, "Experimental evaluation of whole body vibration exposure from tracked excavators with hydraulic breaker attachment in rock breaking operations," Journal of Low Frequency Noise, Vibration and Active Control, vol. 29, no. 2, pp. 101-110, 2010.

[13] D. Lu and Y. Q. Zhou, "Vibration analysis of excavator seat based on EEMD and CWT," Journal of Shandong University (Engineering Science), vol. 45, no. 3, pp. 58-64, 2015.

[14] F. Wang, D. Q. Wang, and Y. Liu, "Hydraulic excavator cab vibration testing and research of vibration reduction," Construction Mechanization, vol. 35, no. 1, pp. 49-51, 2014.

[15] X. K. Pang, Y. Q. Zhou, W. Tang, L. Wang, and Y. Z. Mi, "Excavator seat vibration investigation based on operational transfer path analysis," Journal of Vibration and Shock, vol. 34, no. 9, pp. 171-176, 2015.

[16] Z. Dąbrowski and J. Dziurdź, "Simultaneous analysis of vibrations and noise in the task of minimizing vibroacoustic activity of machines," Archives of Acoustics, vol. 41, no. 2, pp. 303-308, 2016.

[17] Z. J. Li, T. Mao, S. X. Liu, and G. H. Liang, "Dynamic equations of hydraulic excavator slewing transmission mechanism-working device system," Equipment Manufacturing Technology, vol. 19, no. 2, pp. 1-5, 2012.

[18] G. R. Zhang, D. J. Yu, L. Y. Yao, and X. G. Zang, "Study on automotive interior structural noise excited by engines," Noise and Vibration Control, vol. 30, no. 1, pp. 44-47, 2010.

[19] P. Casoli, M. Pastori, F. Scolari, and M. Rundo, "Active pressure ripple control in axial piston pumps through highfrequency swash plate oscillations-A theoretical analysis," Energies, vol. 12, no. 7, p. 1377, 2019.

[20] Z. J. Li, Y. Y. Huang, and Y. Sun, "Effect of the piston pump pressure pulsation on the vibration characteristics of the hydraulic excavator," Chinese Hydraulics \& Pneumatics, vol. 45, no. 2, pp. 77-84, 2021.

[21] J. Chen, F. Qing, and X. Pang, "Mechanism optimal design of backhoe hydraulic excavator working device based on digging paths," Journal of Mechanical Science and Technology, vol. 28, no. 1, pp. 213-222, 2014.

[22] B. V. Chapnik, G. R. Heppler, and J. D. Aplevich, "Modeling impact on a one-link flexible robotic arm," IEEE Transactions on Robotics and Automation, vol. 7, no. 4, pp. 479-488, 1991.

[23] J. F. Jia, J. Q. Zhang, J. Zhang, Y. L. Liu, Y. Q. Gao, and J. Yue, "Ride comfort simulation of tracked vehicles from stochastic excitation based on road surface spectrum," Journal of System Simulation, vol. 24, no. 6, pp. 1350-1354, 2012.

[24] Y. Sun, Study on Vibration Mechanism and Vibration Control Method of Excavator Cab, Guangxi University, Nanning, China, 2018.

[25] A. Bedotti, M. Pastori, and P. Casoli, "Modelling and energy comparison of system layouts for a hydraulic excavator," Energy Procedia, vol. 148, pp. 26-33, 2018.

[26] X. F. Liu, W. Zhang, J. L. Liu, and L. P. Liu, "Design and research on selection for seal of hydraulic system and oil inlet of pipe joint," Chinese Hydraulics \& Pneumatics, vol. 42, no. 12, pp. 97-101, 2018. 
[27] Z. H. Liu, Q. H. Gao, Z. Liu, and X. Wang, "In-plane rigidelastic coupling dynamic modeling and vibration response prediction of heavy duty radial tire," Acta Armamentarii, vol. 39, no. 2, pp. 224-233, 2018.

[28] X. Zhang, X. Li, C. X. Hao et al., "Experimentation on vibration transmission characteristics of modern tram tracks," Journal of Southwest Jiaotong University, vol. 56, no. 1, pp. 75-83, 2021.

[29] G. Y. Ji and C. S. Zhao, "Summary of vibration testing and analysis," Machine Building \& Automation, vol. 39, no. 3, pp. $1-5,2010$.

[30] L. G. Chen, J. G. Wang, and J. Zhang, "Vibration test and signal analysis of RV reducer," Journal of Mechanical Transmission, vol. 42, no. 3, pp. 80-84, 2018. 